\title{
Studying the Mechanisms of Nitro Compounds Reduction (A-Review)
}

\section{R. SASSYKOVA ${ }^{1}$, Y.A. AUBAKIROV ${ }^{1}$, S. SENDILVELAN ${ }^{2}$, ZH.KH.TASHMUKHAMBETOVA ${ }^{1}$, N. K. ZHAKIROVA ${ }^{1}$, M. F. FAIZULLAEVA ${ }^{3}$, A.A. BATYRBAYEVA ${ }^{1}$, R. G. RYSKALIYEVA ${ }^{1}$, B.B.TYUSSYUPOVA ${ }^{1}$ and T.S. ABILDIN ${ }^{1}$}

\author{
${ }^{1} \mathrm{Al}-$ Farabi Kazakh National University, 71, al-Farabi ave., 050040, Almaty, Kazakhstan. \\ ${ }^{2}$ Department of Mechanical Engineering, Dr. M.G.R Educational and Research Institute, \\ University, Chennai, 600 095, India. \\ ${ }^{3}$ Korkyt Ata Kyzylorda University, 29a Aiteke bi str., Kyzylorda, Kazakhstan. \\ *Corresponding author E-mail: larissa.rav@mail.ru \\ http://dx.doi.org/10.13005/ojc/350103
}

(Received: November 26, 2018; Accepted: December 11, 2018)

\section{ABSTRACT}

The article describes some variants of mechanisms of nitro compounds reduction, offered by authors in the scientific literature. The focus is on the description of the work of Kazakh and Russian chemists working in the field of catalysis. In most of the works, the classical scheme of the mechanism of the hydrogenation of Haber-Lukashevich nitrobenzene is confirmed and detailed by experimental methods. One of the interesting aspect of the article is description the mechanism of Ya.A. Dorfman which used the orbital theory of catalysis. The orbital approach of the Ya.A. Dorfman mechanism is constructed in the light of modern orbital representations and is valid for the hydrogenation of the nitro group on various catalysts. Among the discussed mechanisms are ideas of Haber, Debus, Jungers, V.P. Shmonina, Lukashevich, M. Geirovsky, Yu. B. Vasilyev, M. V. Klyuev, E. F. Weinstein, E. Gelder, Ya.A. Dorfman and others.

Keywords: Aromatic nitro compounds, Catalytic reduction, V. P. Shmonina, Haber-Lukashevich, Ya.A. Dorfman.

\section{INTRODUCTION}

The kinetic studies of heterogeneous catalytic reactions are very complex, which is connected with the need to take into account, besides the effect of temperature, the nature of the reagents and solvent, such factors as the nature of the catalyst, carrier, state of the surface of the catalyst. It should also be borne in mind the possibility of diffusion control of the reaction rate.
Therefore, the patterns of influence of conditions on the course of the same reaction when it is carried out on various catalysts can be very different. Apparently, in this connection, the available kinetic data are very contradictory. On their basis, it is impossible to draw definitive conclusions about the influence of the structure of nitroarenes on the rate of their reduction. It is well known that the regularities of the reactions of hydrogenation of substituted nitro compounds are directly related to the staging

This is an Open Access article licensed under a Creative Commons license: Attribution 4.0 International (CC- BY). Published by Oriental Scientific Publishing Company @ 2018

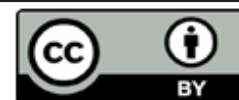


of the nitro group transformations. The complex study of individual stages of the reaction makes it possible to reveal the most probable mechanism of interactions, to determine the reactivity parameters of the initial and intermediate compounds, to form the basis for the mathematical description of the process under study. An important component of this approach is the systematic study of the kinetics of the individual stages of the transformation of the starting materials and reaction intermediates. Obviously, that the use of a comprehensive approach that includes, in addition to kinetic studies, quantum-chemical modeling of reagents, mechanisms and transition states of reactions, makes it possible to understand the features of chemical interaction at the molecular level.

In the literature, various options of conversion schemes for substituted nitro compounds are written. The aim of the article is to discuss some variants of mechanisms of nitro compounds reduction written in scientific literature. The focus is on the description of the work of Kazakh and Russian chemists working in the field of catalysis.

\section{Experimental study of the mechanism of nitro compounds reduction Haber-Lukashevich' mechanism. Ideas of V. P. Shmonina}

The first known mechanism scheme of possible substances formation when the electrochemical reduction of nitrobenzene in the medium of strong bases (strong acids) and in very weakly alkaline or slightly acidic mediums was proposed by Haber in the late $19^{\text {th }}$ century-early $20^{\text {th }}$ century ${ }^{1}$. Another scheme of the catalytic reduction of nitrobenzene on Raney nickel was offered by Debus and Jungers ${ }^{1,2}$. They assumed that azobenzene is formed because of nitrobenzene and amine interaction.

V.P.Shmonina et al., ${ }^{2-4}$ researched reduction of nitrobenzene and different derivatives of it over skeleton nickel and platinum and palladium blacks. Processes were investigated at different amounts of a catalyst, nitro compounds, technological parameters (temperature, solvents, mixing intensity). The scientists studied impact of various substances in the reaction medium (for example, nitrobenzene- aniline intermediates, aniline or acid, pyridine) applying the way of determination of catalyst potential developed by D.V. Sokolsky, V. Drouz ${ }^{3}$ and methods of a polarography ${ }^{4}$ and a conductometric titration. The transformation scheme proposed by them is a development of the well-known mechanism of HaberLukashevich, which described the transformations of a nitro group during its chemical and electrochemical reduction ${ }^{5}$. The Shmonina' mechanism shows transformation of studied nitro compounds(1) to the corresponding amines(4). On the basis of the expirements V. P. Shmonina defined three directions of nitro compounds reduction (Fig.1). This process proceeds through the successive catalytic interaction of the nitro group with three moles of hydrogen by means of formation of intermediate nitro compounds(2) and arylhydroxylamines(3). This first type of conversion, "a so-called hydrogenation direction", is a sequential interaction of a nitro group with three hydrogen molecules. Intermediates by the direction are nitrosobenzene, phenylhydroxylamine, which cannot be collected in the reaction medium, they are immediately converted to aniline as they form. By the second direction according to Shmonina' mechanisms the interaction of nitrobenzene (NB) with phenylhydroxylamine (PHA) and formation of azoxybenzene proceeds, it is then transformed to aniline through azo- and hydrazobenzene. By the third direction $\mathrm{PHA}$ formed because of the reduction of NB than transformed into hydrazobenzene.

So, according to the proposed scheme (Fig. 1), the hydrogenation of NB (1) proceeds through a series of sequential and parallel stages. The catalytic conversion of NB to aniline (A) (4) occurs through the formation of nitrosobenzene (NSB) (2) and PHA (3) to A (4) by successive addition of three hydrogen molecules to the nitro group. Such a mechanism of transformations is called "hydrogenation". At elevated temperatures and elevated pressures of hydrogen, further hydrogenation of $A$ to cyclohexylamine(5) is possible. A distinctive feature of the scheme V.P. Shmonina from the staged transformations proposed by Haber-Lukashevich, is the inclusion of reactions of interaction of intermediate products. These reactions proceed homogeneously in the volume of the solution, according to the so-called "condensation mechanism". 


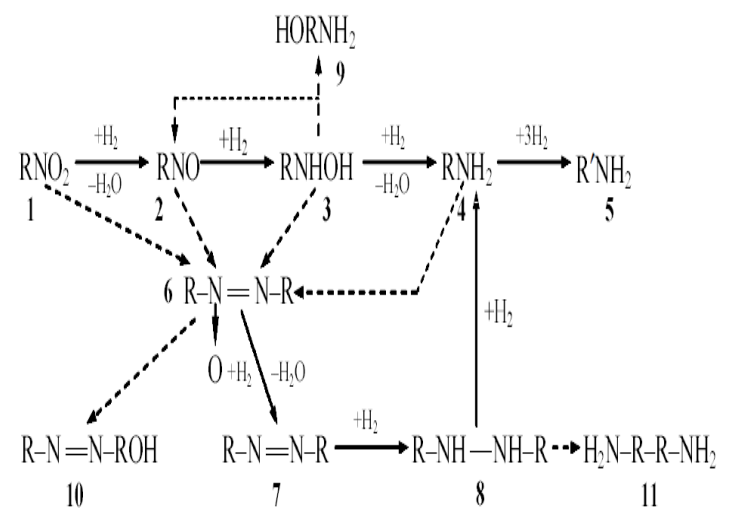

Fig.1. Mechanism proposed by Shmonina ${ }^{6-10}: 1$ - nitrobenzene; 2-nitrosobenzene; 3-phenylhydroxylamine; 4-aniline; 5-cyclohexylamine; 6-azoxybenzene; 7-azobenzene; 8-hydrazobenzene; 9-4-aminophenol; 10-4-hydroxyazobenzene; 11-benzidine

It should be emphasized that nitrosobenzene in the reaction systems is most often not fixed, and is introduced a priori into the schemes of NB transformations as a product preceding the formation of $\mathrm{PHA}^{10-18}$. Probably, the basis for the inclusion of nitrosobenzene in the scheme of NB transformations is the sequence of stages discussed in earlier papers ${ }^{9,10,19}$, since direct evidence of the presence of nitrosobenzene in the solution volume was not given by the authors ${ }^{12,15,16}$. In some works ${ }^{18,20}$, experiments showing formation of nitrosobenzene as an intermediate product are described.

M. Geirovsky 2,20 , in the polarographic reduction of nitrobenzene in aqueous, aqueous-alcoholic and acidic media, found that nitrobenzene joins 4 electrons and is reduced to phenylhydroxylamine, which, further, attaching two electrons at more negative potentials, turns into aniline through azoxybenzene. In an alkaline medium, nitrobenzene attaches 6 electrons at once and, without leaving the cathode, turns into aniline. In an alkaline medium, nitrobenzene attaches 6 electrons at once and, without leaving the cathode, turns into aniline.

The reduction of aromatic nitrocompounds on a supported $3 \% \mathrm{Pt} / \mathrm{SiO}_{2}-\mathrm{AlPO}_{4}$ catalyst was studied in ${ }^{21,22}$. The authors proposed a mechanism for the hydrogenation of aliphatic and aromatic nitro compounds on supported Pt catalysts (Figure 2).

$$
\begin{aligned}
& \mathrm{ArNO}_{2}+\text { metal } \stackrel{1}{\rightarrow} \text { oxidized metal surface }+\mathrm{ArN}:(\mathrm{ads}) \stackrel{\mathrm{ArNO}_{2}}{\rightarrow} \stackrel{\mathrm{I}}{\rightarrow} \text { R-N-O- } \\
& \mathrm{H}_{2} \\
& \text { (dimeric or oligomeric intermediates) } \rightarrow \text { R- } \mathrm{NH}_{2}+\text { metal }+\mathrm{H}_{2}
\end{aligned}
$$

Fig. 2. Mechanism of hydrogenation of aliphatic and aromatic nitro compounds on supported Pt catalysts ${ }^{21,22}$

In this case, the presence of electrondonating groups should slow down the first stage, and, consequently, inhibit the formation of nitrene, thus giving an increase in the lower conversion than nitrobenzene. Conversely, the presence of electron-accepting substituents should stabilize the intermediate nitrene and the rate to stage (2) with respect to nitrobenzene.

In studies ${ }^{23,24}$, upon reduction of chloronitrobenzene to $\mathrm{Ni}$-skeleton, $5 \% \mathrm{Ir} / \mathrm{C}$, the main intermediate of the reaction was chlorophenylhydroxylamine. The adsorptive capacity of the starting compound is higher than that of chlorophenylhydroxylamine, so the authors suggested that, while there is chloronitrobenzene on the surface of the catalyst, phenylhydroxylamine accumulates in the reaction mixture without further conversion to chloroaniline. The authors found that chloraniline forms in the entire range of the investigated concentrations of chloronitrobenzene in the reaction mixture. Based on the analysis of reaction products and hydrogen balance from the gas phase, it was assumed that, together with the hydrogenation direction of reaction (Fig.3) there is a disproportionation of phenylhydroxylamine on the nitroso- and amino compound according to reaction (1):

$$
2 \mathrm{ArNHOH} \leftrightarrow \mathrm{ArNO}+\mathrm{ArNO}_{2}+\mathrm{H}_{2}
$$

The nature of the catalyst, the temperature of the test, the $\mathrm{pH}$ of the medium have a significant effect on the disproportionation rate of phenylhydroxylamine. Catalysts on the activity in this reaction form a series: $\mathrm{Ni}<\mathrm{Ir}<\mathrm{Pd}=\mathrm{Pt}$.

Yu. B. Vasilyev ${ }^{25,26}$ proposed a mechanism for the hydrogenation of nitro compounds on $\mathrm{Pt}$, 
proceeding along the hydrogenation pathway. The reaction can proceed via the O-Pt or N-Pt bond with the desorption of products of incomplete reduction of the NB into the volume (Figure 4).

$$
\mathrm{ArNO}_{2} \stackrel{\mathrm{H}_{2}}{\rightarrow} \text { ArNO } \stackrel{\mathrm{H}_{2}}{\rightarrow} \text { ArNHOH } \stackrel{\mathrm{H}_{2}}{\rightarrow} \mathrm{ArNH}_{2}
$$

Fig. 3. The hydrogenation direction of a reaction ${ }^{23,24}$

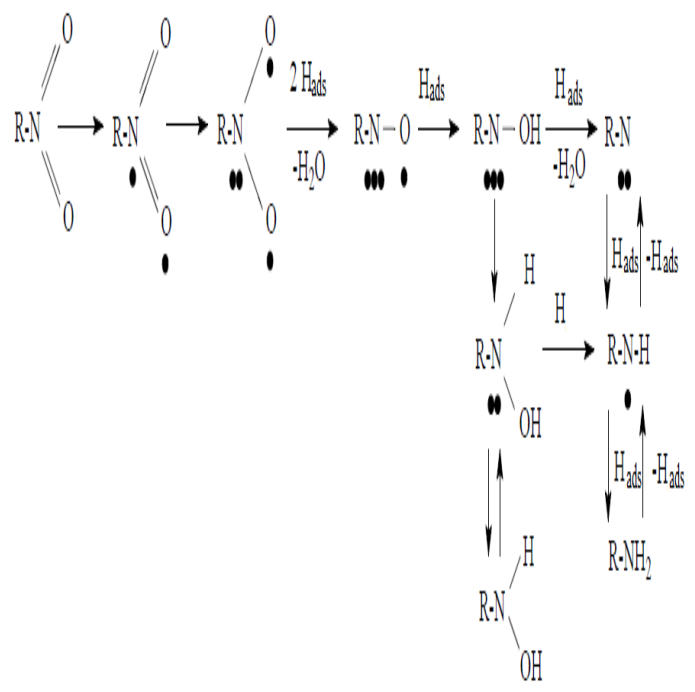

Fig. 4. Hydrogenation scheme of nitrobenzene on platinum, proposed by Yu.B. Vasilyev

M.V. Klyuev and E.F. Weinstein in a paper ${ }^{27}$ assumed that in a liquid-phase hydrogenation of nitroarenes on palladium-polymer catalysts, the substrate interacts with catalyst metal centers containing activated hydrogen. The resulting adduct "collects" the missing hydrogen from the surface of the catalyst. Then hydrogen displaces the formed amine into the solution.

It was found in the synthesis of anesthesin by liquid phase hydrogenation of ethyl 4-nitrobenzoic acid on palladium-based catalysts, for example $\mathrm{Pd} / \mathrm{C}$, that the reaction proceeds without the formation of intermediate products ${ }^{28}$, which corresponds to the hydrogenation pathway in the Haber-Lukashevich scheme. In the case of nitrobenzene hydrogenation on a $\mathrm{Pd} / \mathrm{C}$ catalyst in methanol and 2-propanol, only NB and AN were detected in the reaction mixture. The authors assume that in different solvents the reaction takes place at various active centers of the $\mathrm{Pd}$ surface, and as a result, the transition from methanol to 2-propanol changes the limiting stage from the hydrogenation of NB to the hydrogenation of nitrosobenzene.

Extremely interesting are the data obtained in studying the kinetics of the hydrogenation reactions of isomers of halogen-substituted nitrobenzenes on palladium supported on coal or functional polymers in various solvents such as cyclohexane, methanol, diethyl ether, dioxane and tetrahydrofuran ${ }^{29,30}$. Despite the fact that the authors used various methods of analytical control, in particular, gas chromatography and NMR spectroscopy, condensation products: azoxy, azo and hydrazo substituted - were not detected by them. This fact can indirectly indicate that an alternative way of obtaining an amine is possible, which excludes the formation of such intermediate compounds as nitrosobenzene and phenylhydroxylamine.

\section{E. Gelder' scheme}

E. Gelder' scheme ${ }^{31-33}$ is shown on Fig.5. The author rejects the traditional mechanism of reaction offered by Haber and Lukashevich and offers the alternative scheme which, according to them, is fair for other metallic catalysts. According to this scheme an intermediate product of transformation of nitrogroup is the semi-hydrogenated form$\mathrm{C}_{6} \mathrm{H}_{5}(\mathrm{OH})$ which participates in all subsequent stages. Thus, during its catalytic interaction with hydrogen azobenzene (AB) or phenylhydroxylamine is formed (PHA), and during interaction of these forms azoxybenzene (AOB) is appeared.

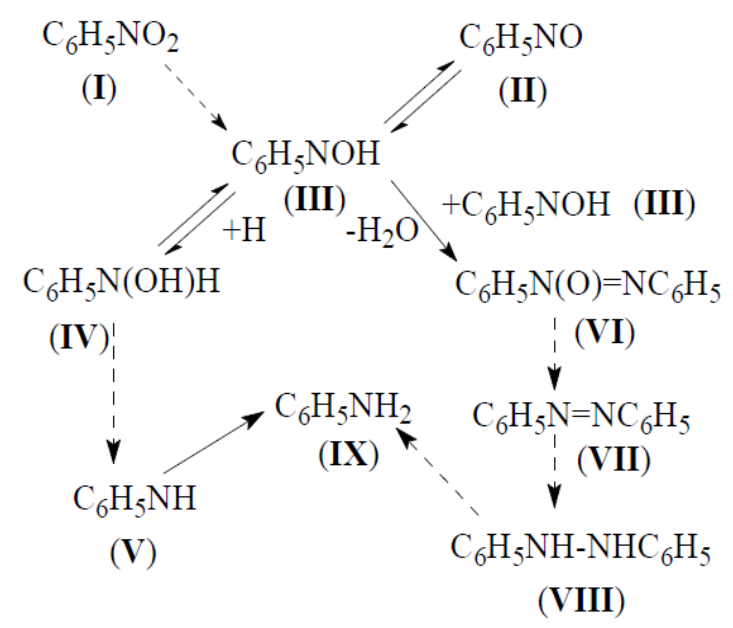

Fig. 5. E. Gelder' scheme 
The author assumed that on the surface of the catalyst there is the general intermediate compound of NB and a nitrozobenzene (III) (Fig. 5) which in case of hydrogenation of NB reacts with the adsorbed hydrogen, consistently turning into PHA and AN through intermediate V. Hydrogenation of a nitrozobenzene reacts with 2 molecules of intermediate compound with removal of a molecule of water and formation of azoxybenzene (AOB) which then turns into $A B$, hydrazobenzene $(H B)$ and aniline.

Thus, in most works, the classical scheme of the hydrogenation of nitrobenzene HaberLukashevich is confirmed and detailed by various experimental methods. However, there are alternative judgments, as the E. Gelder' scheme.

For example, in a case of $\mathrm{Au} / \mathrm{TiO}_{2}$ catalyst $^{34}$ is suppressed an accumulation of condensation products when hydrogenation of aromatic nitro compounds. It was found that nitrosobenzene is appeared in the reaction mixture in very small amounts; hydroxylamine and nitrosobenzene interact strongly with the catalyst surface. On the base of numerous experiments the authors proposed a new reaction mechanism for the reaction (Figure 6).

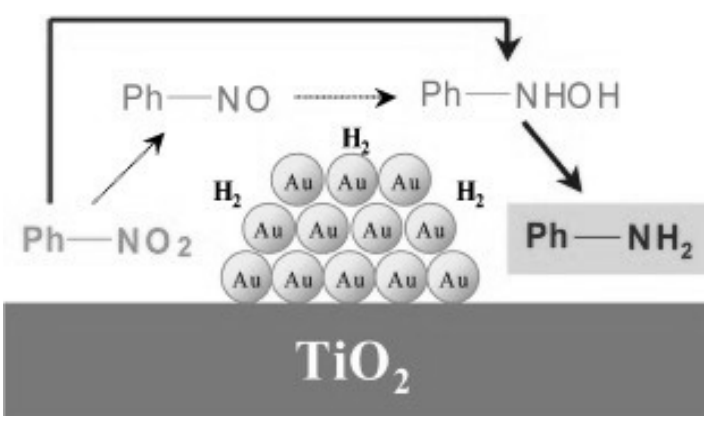

Fig. 6. A new mechanism of aromatic nitro compounds hydrogenation over $\mathrm{Au} / \mathrm{TiO}_{2}$ catalyst

The complexity of studying the mechanism of this reaction can be explained by the multistage nature of the reduction of the nitro group (Fig.5), as well as the influence on the rate of hydrogenation of a number of factors.

Most likely, analyzing the literature data suggest that the hydrogenation of nitrobenzene proceeds in accordance with the scheme of HaberLukashevich, regardless of the nature of the catalyst, solvent and nitro substituents in the molecule. However, the change in catalyst and solvent significantly affects the ratio of the rates of individual heterogeneous catalytic and homogeneous stages, changing their contribution to the overall reaction rate. Therefore, the mechanism of hydrogenation of nitro compounds on different catalysts requires additional studies. In particular, for the classical $\mathrm{Pd} / \mathrm{C}$ hydrogenation catalyst, which can be used in the hydrogenation acylation of nitroarenes, the mechanism has not been specified ${ }^{32}$.

\section{Ideas on the basis of experimental data and quantum-chemical calculations}

Analysis of experimental data and quantumchemical calculations in in $^{32,35-37}$ shows that the addition of $\mathrm{H} \delta$ - to the NB molecule reduces charges on oxygen atoms and increases the charge on the nitrogen atom. The charge on the nitrogen and oxygen atoms of nitrosobenzene $(\mathrm{H} \delta$-) is significantly reduced in comparison with nitrosobenzene.

The charge on all the atoms of the reaction center of the reaction product of $\mathrm{PHA}$ with $\mathrm{H} \delta$ - is substantially higher than that of the initial PHA; only oxygen atom remains negatively charged. Azoxybenzene $(\mathrm{AOB})(\mathrm{H} \delta-)$, in contrast to the $\mathrm{AOB}$ molecule, carries a negative charge on both nitrogen atoms, and the negative charge on the oxygen atom is somewhat smaller in comparison with the AOB molecule.

Finally, the product of the interaction of the $A B$ molecule with the first hydrogen atom carries a positive charge on both nitrogen atoms, unlike the $A B$ molecule, which has a negative charge on nitrogen atoms. On the basis of the foregoing, the authors proposed a mechanism for the hydrogenation of NB on a Pd/C catalyst (Fig.7) 37,38 . The mechanism is based on the results of quantum-chemical modeling of nitrobenzene (NB) molecules, intermediates of its reduction, and products of the addition of the first hydrogen atom to these substrates, as well as the following experimental facts:

1) at hydrogenation of NB on the $P d / C$ catalyst in a reaction mixture at any stage of transformation nitrosobenzene, phenylhydroxylamine, $A O B$, $A B$ and a hydroxylamine are not found. In solution there is only aniline ${ }^{2,37}$.

2) at hydrogenation of nitroarenes and intermediates of reduction of NB the kinetic constants of reactions depend on a charge 
on the reactionary center $2,35,38$.

3) nitroarenes are hydrogenated by strongly bound hydrogen ${ }^{35-38}$ at activation on palladium clusters hydrogen can acquire both a positive and a negative charge $\mathrm{e}^{38-41}$.

4) nitroarenes displace hydrogen $n^{2,38,39}$ from the surface of palladium;

5) nitroarenes are adsorbed on the catalyst by nitrogen and oxygen atoms of the nitro group ${ }^{25-27,42}$ and create a positive charge on its surface ${ }^{39,43}$ palladium adsorbed on coal is also positively charged ${ }^{30}$.

6) the process of catalytic hydrogenation of nitroarenes is limited by a chemical act on the catalyst surface, while the rate of the determining step is the addition of the first hydrogen atom ${ }^{2,38}$.

Although the hydrogenation pathway for the reduction of NB to $\mathrm{Pd} / \mathrm{C}$ has been established in $^{32,36,37}$, changing the conditions, for example, the solvent, $\mathrm{pH}$, or the addition of additives, for example inorganic salts ${ }^{2,32}$, can lead to a change in the ratio of the rates of the individual hydrogenation steps and the realization condensation direction. In this connection, both routes of reaction are considered in the proposed mechanism.
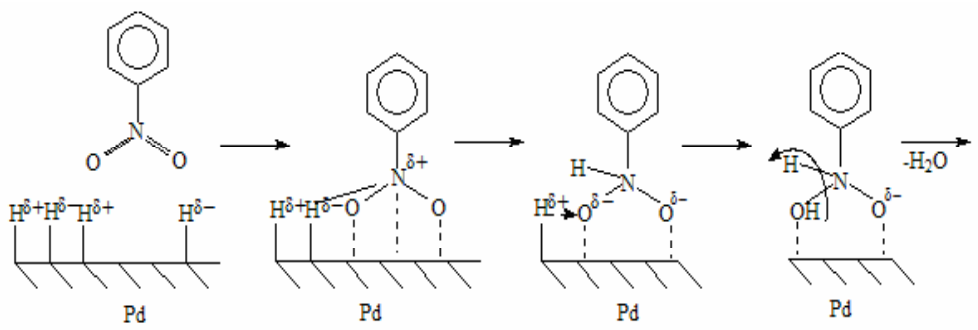

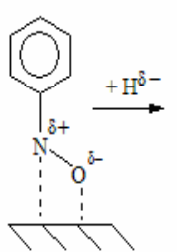

$\mathrm{Pd}$

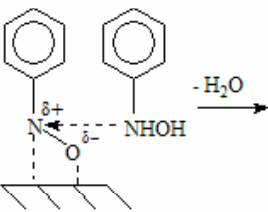

$\mathrm{Pd}$

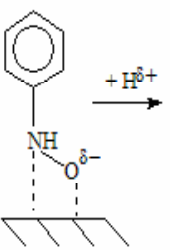

$\mathrm{Pd}$

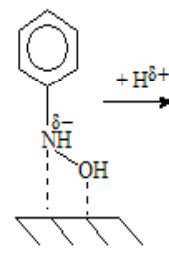

$\mathrm{Pd}$

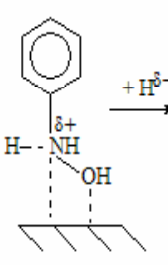

$\mathrm{Pd}$
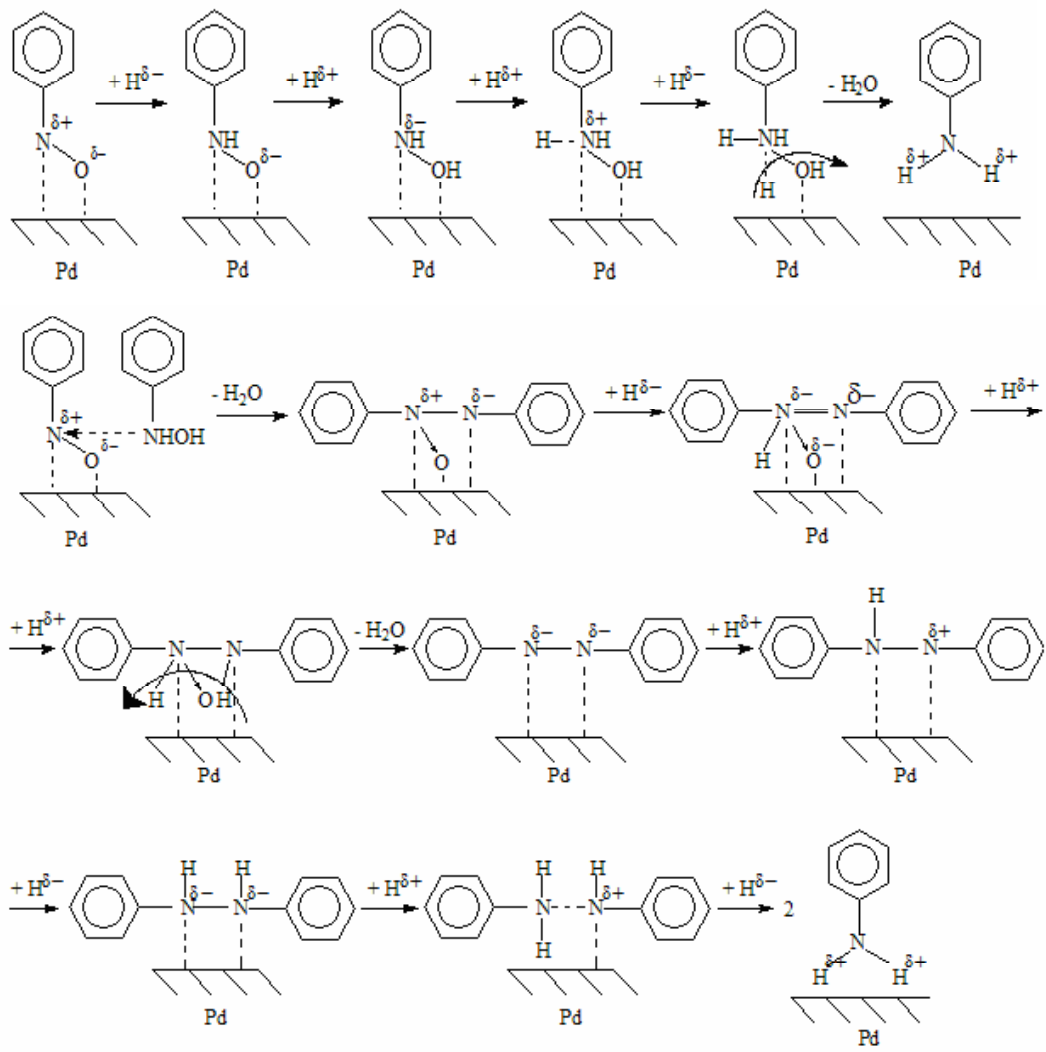

Fig. 7. The mechanism of hydrogenation of NB on a Pd/C catalyst based on experimental data and quantum chemical calculations: a-hydrogenation direction, b-condensation direction ${ }^{27}$ 
This mechanism is also applicable to the related hydrogenation of ethyl 4-nitrobenzoic acid ester used in the synthesis of anesthesin (Fig.8). This reaction also proceeds in accordance with the scheme of Haber-Lukashevich.

The nature and composition of the solvent have a significant effect on the speed of all stages of chemical transformations of functional groups, changing the ratio of the contributions of the hydrogenation direction and condensation interactions to the overall reaction rate. Thus, hydrogenation of nitrobenzene on platinum, palladium and nickel in aqueous solutions of aliphatic alcohols at atmospheric pressure of hydrogen takes place selectively until the formation of aniline, the condensation products of intermediate products in the reaction medium are completely absent ${ }^{2,6,8,38}$.

Introduction to water-alcohol solutions of $20 \%$ by wt. pyridine having a high electron-donor ability increases the yield of hydrazobenzene to values close to quantitative ${ }^{44}$, and if the reaction is carried out in the presence of additives of sodium hydroxide on the supported catalyst, for example $\mathrm{Pd} / \mathrm{C}$, then the yield of hydrazobenzene exceeds $80 \%{ }^{44}$.

In connection with the wide practical application of halogenoanilines, the mechanism of liquid-phase hydrogenation of halogen-substituted nitrobenzenes is of particular interest. Along with the

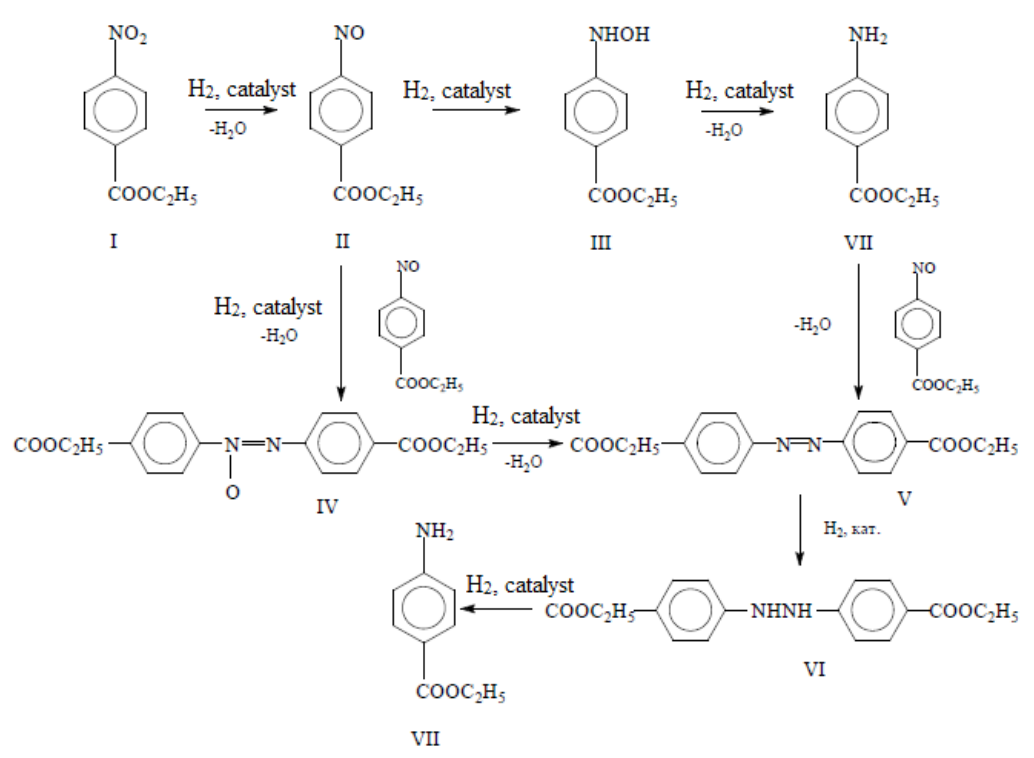

Fig. 8. The mechanism of hydrogenation of ethyl 4-nitrobenzoic acid ${ }^{32}$ catalytic transformations of the nitro group, shown by V.P. Shmonina in Fig.1, in most cases secondary processes of dehalogenation occur $2,32,38,44$.

The halogens are cleaved mainly from the molecules of substituted aminobenzenes, interacting with hydrogen by the catalytic mechanism ${ }^{4-46}$, and the degree of dehalogenation essentially depends on the nature of the catalyst $2,32,38,44-46$. Thus, the rate of dechlorination during the hydrogenation of 2-nitrochlorobenzene on a skeletal nickel catalyst in aqueous solutions is quite high. The yield of 2 -aminochlorobenzene does not exceed $20 \%$, and when iridium catalysts are used, the degree of dehalogenation is $0.05-0.10 \%{ }^{44}$.

Thus, the optimum catalytic system for liquid-phase hydrogenation reactions of halogensubstituted nitrobenzenes should provide not only a high degree of conversion of the nitro group, but also a low degree of dehalogenation.

Data available in the literature on the mechanism of catalytic conversions of azo group are extremely limited. Most often during the hydrogenation, substituted azobenzene, successively adding two moles of hydrogen and then - are converted into the corresponding amines ${ }^{2,6-8,38}$. In general, such a mechanism is consistent with the chemical transformations of the intermediate products - substituted azoxy- and azobenzenes, shown in Figure 1.

III 
A certain analogy is noted in the kinetic regularities of the reactions of liquid-phase hydrogenation of the azogroup and the $-\mathrm{C}=\mathrm{C}$-bond in the alkene derivatives ${ }^{2,6-8,38}$.

Examples of the effect of the nature and composition of the solvent on the rate and selectivity of reactions involving not only substituted nitrobenzenes but also azobenzenes are given $\mathrm{in}^{27}$, 47,48. Chemical transformations in the reactions of liquid-phase hydrogenation of compounds whose molecules contain several functional groups capable of participating in the catalytic process are substantially complicated ${ }^{2,6-8,38}$. Each of the groups, having a different reactivity with respect to hydrogen, makes an unequal contribution to the overall rate of hydrogenation $^{49,50}$.

As for the stoichiometric mechanism of such reactions, most often it cannot be represented as a simple set of stages of chemical transformations of individual functional groups: there is the possibility of steric hindrance, which reduces the rate of their interaction with hydrogen, the probability of side condensation of intermediate products, intramolecular regrouping and cyclization, which leads to the formation of various compounds ${ }^{51-60}$.

It can be assumed that the kinetic parameters of the reactions of liquid-phase hydrogenation of compounds with several reactive groups, primarily selectivity, are most sensitive to the properties of the catalytic system and to changes in the conditions of the process.

The hydrogenation of one of the most wellknown classes of compounds with various reactive groups - substituted 2-hydroxynitroazobenzenes - was studied in ${ }^{60,61}$. Fig. 9 shows the scheme of chemical transformations of 2-nitro-2'-hydroxy-5'methylazobenzene during liquid-phase hydrogenation. Substituted 2-hydroxynitroazobenzenes are the starting materials for the preparation of compounds of the class $2 \mathrm{H}$-benzotriazoles, which are effective photostabilizers of polymeric materials. The authors have found that, regardless of the nature of the catalyst and the solvent, in reaction with hydrogen participate simultaneously both nitro and 2-substituted azo hydroxynitroazobenzenes. At the initial stage of the reaction, nitroso- and nitrohydrazo derivatives (products 2, 5 in the scheme of Fig. 9) are formed. Further hydrogenation of the substituted 2-nitrosoazobenzene proceeds through the hydroxylamine derivative (3) to form the aminoazo derivative (4), which then in the presence of the catalyst is converted to 1,4-phenylenediamine and 2-hydroxy-5-methylaniline (15), (16) via the amino hydrazo derivative (6). The hydrazo group of substituted 2-nitrohydrazobenzene (5) catalytically decomposes to form 4-nitroaniline and 2-hydroxy-5-methylaniline (14), (16). Further, 4-nitroaniline, reacting with hydrogen, is converted to 1,4-phenylenediamine (15). Some intermediate products, in particular, (2), (3) and (9), are localized only in the surface layer of the catalyst, and their concentrations in solution are either absent or very small.

The main feature of the hydrogenation of substituted 2-hydroxynitroazobenzenes is that the intermediate products of the nitro and azo group conversions are capable of forming a triazole ring, the rearrangement of the substituted 2-nitrosoazobenzene occurs in the surface layer of the catalyst and 2-nitrohydrazobenzene is homogeneous in the volume of the solution. During the triazole rearrangement, the $\mathrm{N}$-oxide of substituted benzotriazole (7) is formed in the reaction medium, which, catalytically reacting with hydrogen, is converted to substituted $2 \mathrm{H}$ benzotriazole (8). When the reaction is carried out at high hydrogen pressures, low concentrations of 2-hydroxynitroazobenzene and a high catalyst content, further hydrogenation of the $-\mathrm{C} \equiv \mathrm{C}$ - and $-\mathrm{C} \equiv \mathrm{N}$-bonds in the molecules of substituted $2 \mathrm{H}$-benzotriazoles is possible with the formation of di-, tetra-, hexa- and octahydrodroducts (products (10), (11), (12) and (13).

Information on the study of the mechanism of reduction of di- and poly-nitrocompounds is very insignificant. For example, the hydrogenation of di- and trinitrobenzanilides on a $\mathrm{Pd} / \mathrm{C}$ catalyst in dimethylformamide at 5. $0 \mathrm{MPa}$ and $333 \mathrm{~K}$ was studied $\mathrm{in}^{60,61}$. It was shown that when hydrogenation of 2', 4-dinitrobenzanilide of nitro group at position 2', and in 4, 4'-dinitrobenzanilide at position 4' reduces to form, respectively, 2'-amino-4-nitro and 4-amino4'-nitrobenzanilides.

Depending on the conversion in the catalyst, the starting, intermediate and final products are present. The reduction of 2', 4', 4-trinitrobenzanilide 
was also studied. It was found that the most reactive nitro groups are at positions 2' and 4 . In fact, in the reduction of the 4'-nitro group, the assumption was made of the preferential adsorption of 2', 4', 4-trinitrobenzanilide on the catalytic surface by 2 ' and 4-nitro groups.

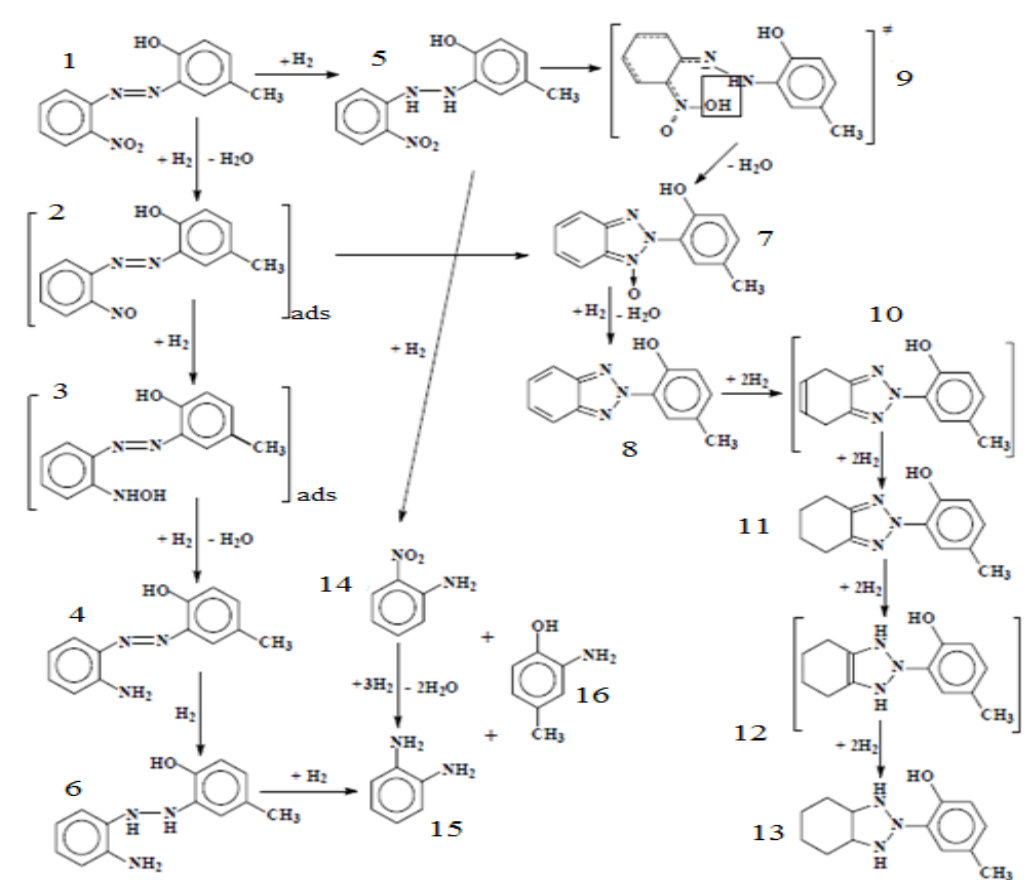

Fig. 9. Chemical transformations of 2-nitro-2'-hydroxy-5'-methylazobenzene in liquid-phase hydrogenation: Ar=2'-hydroxy5'-methylazobenzene; Ar'=2'-hydroxy-5'-methylhydrazobenzene; 1-2-nitro-Ar; 2-2-nitroso-Ar; 3- 2-hydroxylamine-Ar; 4-2amino-Ar; 5-2-nitro-Ar'; 6-2-amino-Ar'; 7-N-oxide-2'-hydroxy-5'-methylphenyl benzotriazole; 8-2'-hydroxy-5'-methylphenylbenzotriazole; 9 - transition state of the product 5; 10-13 di-, tetra, hexa, octahydro-derivatives of 2'-hydroxy-5'methylphenylbenzotriazole; 14 - 2-nitroaniline; 15-1, 4-phenylenediamine; 16-2-hydroxy-5-methylaniline.

In $^{61}$ the mechanism of liquid-phase hydrogenation of aromatic nitrocompounds on palladium-containing polymers is considered. The author believes that the hydrogenation process begins with the interaction of molecular hydrogen with the catalyst metal centers, then the molecules of the nitro compounds interact with metal centers containing activated hydrogen. The resulting adduct is reduced in turn by hydrogen adsorbed on the surface of the catalyst, after which the resulting amine is displaced into the solution by molecular hydrogen (Fig.10). Based on the fact that the degree of filling the "surface" of the catalyst with reagents does not affect the process speed, because the concentration of hydrogen and nitro compound is many times greater than the concentration of active sites, the author believes that the rate of hydrogenation is determined by the "pushing" of the amine molecule into the solution.

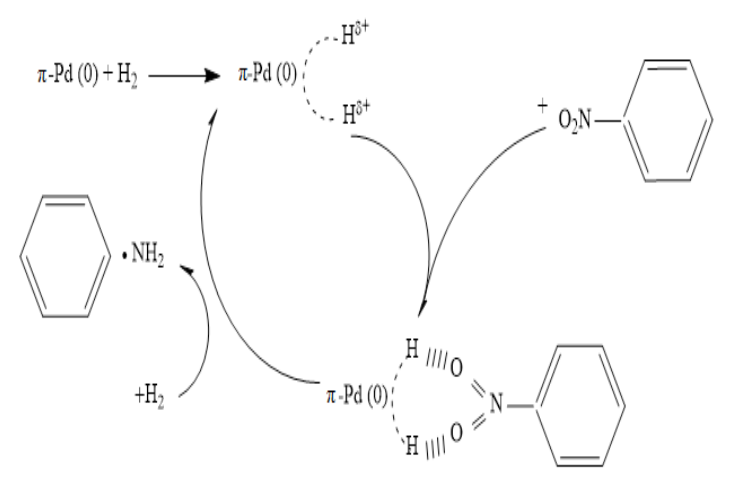

Fig.10. Mechanism of liquid-phase hydrogenation of aromatic nitrocompounds on palladium-containing

The hydrogenation of more than 10 aromatic mono- and dinitrocompounds on $\mathrm{Al}_{2} \mathrm{O}_{3}$-supported catalysts based on platinum-group metals in various solvents under increased hydrogen pressure was studied in $2,38,52,53,62-64$. The effect of the structure and position of substituents on the aromatic ring on the kinetics and mechanism of reduction of aromatic 
nitro compounds over $4 \% \mathrm{Pd} / \mathrm{Al}_{2} \mathrm{O}_{3}$ was studied. It is shown that the aromatic nitro compounds studied are reduced in the hydrogenation direction without accumulation of products of isomerization and hydrogenolysis. Hydrogenated aromatic nitrocompounds, by decreasing the rate of reduction, form a series:

p-nitrophenol>0-nitrophenol> p-nitroaniline > m-nitroaniline> o-nitroaniline>; 2,5-dinitrophenol> 2,4-dinitrophenol> 2,6-dinitrophenol (Figure 11).

A rectilinear relationship between the rate constants and the constants of Hammett substituents was revealed. The experiments confirmed that electron-donor substituents reduce the rate of reduction of nitro compounds.

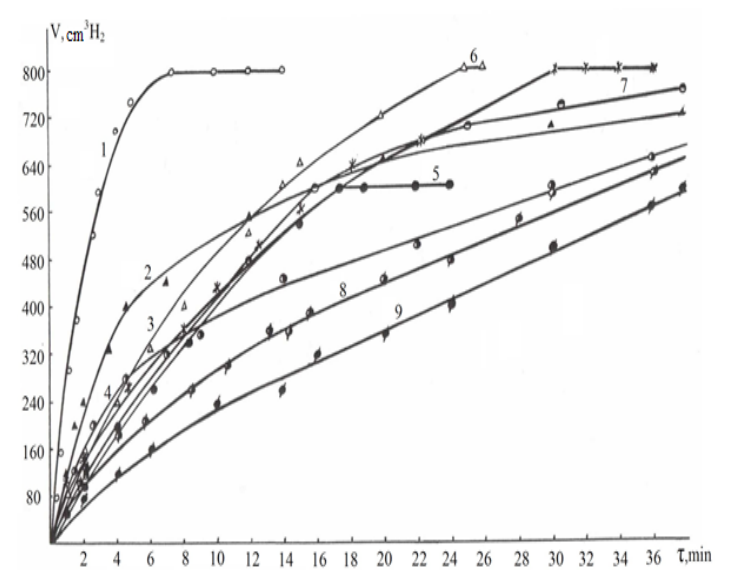

Fig. 11. Kinetic curves for the reduction of various nitro compounds over $4 \% \mathrm{Pd} / \mathrm{Al}_{2} \mathrm{O}_{3}$ at $\mathrm{pH} 2=1.0 \mathrm{~m} . \mathrm{pa}, \mathrm{T}=293 \mathrm{~K}$, $A_{3(6) \mathrm{H} 2}=800 \mathrm{~cm}^{3}$, amount of a catalyst, $q=0.1 \mathrm{~g}$ : 1-nitrobenzene, 2 - 2,5-dinitrophenol, 3-p-nitrophenol, 4-2,6-dinitrophenol, 5-o-nitrophenol, 6-p-nitroaniline, 7-2,4-dinitrophenol, 8- m-nitroaniline, 9- o-nitroaniline

The reduction of twelve $0-, \mathrm{m}$ - and p-substituted nitrobenzene derivatives at atmospheric pressure and room temperature was studied in ${ }^{65,66}$. As catalysts, the authors used Ni-skeleton and Ni-skeleton, modified with Pt.

It is established that the chemical nature and position of the substituents on the aromatic nucleus influence the kinetics of the process, the hydrogenated compounds can be written down in terms of the rate constants: $\mathrm{p}-\mathrm{CHO}>\mathrm{m}-\mathrm{Cl}>\mathrm{O}-\mathrm{OH}$ $>\mathrm{m}-\mathrm{CHO}>\mathrm{o}-\mathrm{NH}_{2}>\mathrm{p}-\mathrm{OC}_{2} \mathrm{H}_{5}>\mathrm{m}-\mathrm{OH}>\mathrm{m}-\mathrm{CH}_{3}>$ $\mathrm{H}>\mathrm{m}-\mathrm{NH}_{2}>\mathrm{p}-\mathrm{CH}_{3}>\mathrm{p}-\mathrm{OH}$.
The $\mathrm{p}$ - and $\mathrm{m}$-substituents in this series, with the exception of $\mathrm{p}-\mathrm{OC}_{2} \mathrm{H}_{5}$, are located in the series shown according to the values of the Hammett substituent constants in decreasing order.

$\ln ^{66}$, when the nitrobenzene, o-nitroanisole, o-nitrophenol, dinitrotoluene, and p-nitrophenol were reduced at atmospheric pressure, it was found that the apparent activation energies are very different and are in the range 20.1-36. $0 \mathrm{~kJ} /$ mol. The highest adsorption capacity was observed for o-nitrophenol. The authors believe that a rather large spread in the values of apparent activation energies is associated with the structure of nitro compounds and the influence of substituents on the electron density in the aromatic nucleus. The reduction of nitroalkylphenols at room temperature and atmospheric pressure was studied in ${ }^{65,66}$. It is shown that the reduction rate decreases with increasing size and complication of the structure of the substituents, which is explained by the authors of the presence of spatial difficulties. In the works $2,38,65-69$, in the study of the hydrogenation of nitrobenzene, p-nitrophenol, $p$-nitrotoluene, $p$-nitrochlorobenzene, $p$-nitroaniline in various solvents on colloidal $R h$ and $\mathrm{Pd}$ at atmospheric pressure, it is noted that the type of substituent affects the reaction rate in those cases, when the order of the reaction on the substrate is of the first or fractional order. If, on the other hand, the reaction order on the hydrogenated nitro compound is zero, then the substituents do not influence the reaction rates and the duration of the process.

$1 \mathrm{n}^{70}$ the hydrogenation of $\mathrm{m}$ - and p-substituted nitrobenzenes in synthesized from colloidal rhodium and palladium catalytic systems supported on polyvinyl alcohol was investigated. The process was studied at atmospheric pressure and ambient temperature. It was found that the reaction order for the nitro compound on both catalysts is zero. The rate of reduction of para-derivatives is reduced in the series.

$$
\begin{aligned}
& \mathrm{NO}_{2} \mathrm{C}_{6} \mathrm{H}_{4} \mathrm{CN}>\mathrm{NO}_{2} \mathrm{C}_{6} \mathrm{H}_{4} \mathrm{CHO}>\left(\mathrm{NO}_{2}\right)_{2} \mathrm{C}_{6} \mathrm{H}_{4}>\mathrm{NO}_{2} \mathrm{C}_{6} \mathrm{H}_{4} \mathrm{C} \\
& \mathrm{OOH}>\mathrm{NO}_{2} \mathrm{C}_{6} \mathrm{H}_{4} \mathrm{l}>\mathrm{NO}_{2} \mathrm{C}_{6} \mathrm{H}_{4} \mathrm{Cl}>\mathrm{NO}_{2} \mathrm{C}_{6} \mathrm{H}_{4} \mathrm{Br}_{6}>\mathrm{C}_{6} \mathrm{H}_{5} \mathrm{NO}_{2}> \\
& \mathrm{NO}_{2} \mathrm{C}_{6} \mathrm{H}_{4} \mathrm{OCH}_{3}>\mathrm{NO}_{2} \mathrm{C}_{6} \mathrm{H}_{4} \mathrm{NH}_{2}
\end{aligned}
$$

For m-substituents authors observed an other series of reducing reaction rate.

$$
\begin{aligned}
& \mathrm{NO}_{2} \mathrm{C}_{6} \mathrm{H}_{4} \mathrm{Cl}>\mathrm{NO}_{2} \mathrm{C}_{6} \mathrm{H}_{4} \mathrm{Br}>\mathrm{NO}_{2} \mathrm{C}_{6} \mathrm{H}_{4} \mathrm{CHO}> \\
& \mathrm{C}_{6} \mathrm{H}_{5} \mathrm{NO}_{2}>\mathrm{NO}_{2} \mathrm{C}_{6} \mathrm{H}_{4} \mathrm{COOH}>\mathrm{NO}_{2} \mathrm{C}_{6} \mathrm{H}_{4} \mathrm{NH}_{2}
\end{aligned}
$$


The authors of the study showed an increase in the reduction rate of compounds having substituent groups attracting electrons and, conversely, a decrease in the reaction rate in the case of hydrogenation of nitro compounds with electron-repelling substituents. Halogen-substituted nitro compound with a m-position substituents is reduced on a rhodium catalyst with on the rhodium highest speed. By the opinion of the authors, on the rhodium catalyst is the process due to the presence of ionized hydrogen, and on the palladium catalyst with the participation of hydrogen atoms. The authors provide a stepwise mechanism for the reduction of nitrobenzene and support their conclusions with data on the electronic configurations of $\mathrm{Pd}$ and $\mathrm{Rh}$ atoms and the difference in the nature of hydrogen adsorption with palladium and rhodium.

In the hydrogenation of various aromatic nitrocompounds in ethanol at atmospheric pressure in the presence of Ru-catalyst reaction rate decreases in the order ${ }^{71}$ :

p-nitrobenzonitrile> p-nitrobenzoic acids $p$-nitrophenol> $p$-chloronitrobenzene $>$ nitrobenzene, $p$-nitroanisole, $p$-nitroaniline, $p$ - nitrotoluene, p-bromonitrobenzene

\section{The mechanism of catalytic reduction of aromatic} nitro compounds created by Ya.A. Dorfman

The orbital approach to the Ya.A. Dorfman mechanism is constructed in the light of modern orbital representations and is valid for the hydrogenation of the nitro group on various catalysts.

The mechanism proposed by Ya.A. Dorfman $^{43}$, is considered using the orbital theory of catalysis. The most important factors, according to the author, are the electronic configurations and energies of the free orbital and the occupied orbitals of the ground state of the active catalyst, the $\sigma$ - and $\pi$-acceptor and $\sigma$ - and $\pi$-donor capacity of substituents, the $\mathrm{pH}$ of the medium, which regulate the direction of chemical transformations of nitro compounds.

In accordance with the mechanism proposed by Ya. A. Dorfman, the catalytic reduction cycle of nitro compounds consists of the following stages:
- dissociation of hydrogen.

- hydrogenation - $\mathrm{NO}_{2}$, - NO-groups.

- hydrolysis of $\mathrm{HO}-\mathrm{NH}$.

Ya.A. Dorfman assumed that nitro compounds attach three molecules along three main routes. Nitro compounds and intermediates have a polar bond of $\mathrm{N}-\mathrm{O}$ and the effect of the catalyst depends on the method of substrate coordination. The first two routes ( $\pi$-route and o-route) differ in the way of coordination of nitro compounds and intermediates with the catalyst.

The first stage of hydrogenation is the activation of hydrogen. The condition for the activation of hydrogen is the ability of the metal to form $\sigma$-bonds with the hydrogen atom, since the latter does not form other bonds. This condition is satisfied both by copper and palladium.

The next stage is the activation of the nitro group. Nitro compounds can be adsorbed and hydrogenated on three routes:

- $\quad$ The $1^{\text {st }}$ way is by means of $\pi$-bonds of the nitro group,

- $\quad$ The $2^{\text {nd }}$ way is through the oxygen atom,

- $\quad 3^{\text {rd }}$ path through the interaction of intermediates with the formation of dimers - azo- and diazo-, which occurs in alkaline media.

The first path ( $\pi$-route) is characteristic for contacts that activate nitro compounds and intermediates by $\pi$-coordination.

The second mechanism (o-route) hydrogenates nitro compounds and intermediates on catalysts preferring o-coordination. The process on both routes starts with the activation of hydrogen and nitro compounds. The interaction between them leads to the accumulation of nitrosobenzene which is hydrogenated to phenylhydroxylamine and aniline.

The share of the third route, apparently, grows in an alkaline environment. Hydroxyl ions catalyze the reaction between intermediates (nitrosobenzene) and aniline, leading to the formation of azobenzene, which is then reduced to hydrazobenzene and aniline.

The first route, like the other two, originates from the stage of hydrogen activation. 
Hydrogenation begins with the stage of hydrogen activation, as a result, atomic hydrogen is formed on the surface. To convert $\mathrm{NO}_{2}$ to $\mathrm{NH}_{2}$, three hydrogen molecules are needed.

The reduction of the nitro group proceeds according to the scheme (Figure 12):

\section{The orbital theory of catalysis introduces three conditions to hydrogen activators}

Oxidative addition of the hydrogen molecule will easily proceed on catalysts with electronegativity of 1.7-2.2, and low energy of promoting of the $p_{2}{ }^{-}$ state. The third condition is the ability to form $\sigma$-bonds with hydrogen atoms, because hydrogen does not form other bonds. These conditions can withstand mainly the elements of the VIII-group of the Periodic Table of D.I. Mendeleev.

On the first route the metals, activating nitro compounds and products using the $\pi$-bonds, are active to hydrogenate. The second route (o-route) is realized on catalysts interacting with nitro compounds and intermediates through an oxygen atom. The third route can be in alkaline media on low-activity catalysts. As a result, the intermediates can react with each other, and the catalyst already hydrogenates the condensation products.

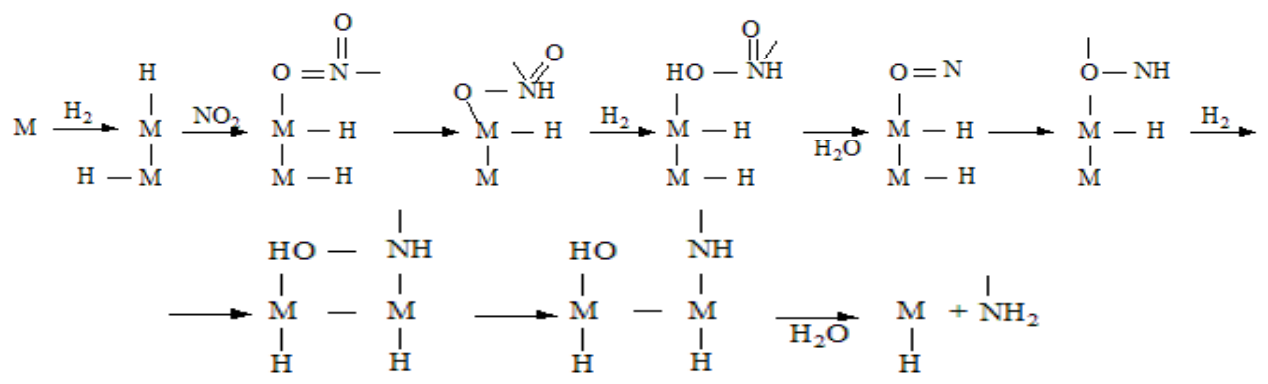

Fig.12. Mechanism of catalytic reduction of aromatic nitrocompounds by Dorfman

The next selection of catalysts is made by the second stage, in which the nitro group is activated. The order of the $\mathrm{N}-\mathrm{O}$ bond and the $\mathrm{O}=\mathrm{N}$ $=\mathrm{O}$ bond angle depends on the way the nitro group interacts with the catalyst.

An analysis of the electronic structure of the nitro group shows that the nitro compounds can be coordinated with the catalyst both with the help of the $\pi$-bond and with the participation of the oxygen atom. The largest negative charge is localized on oxygen atoms. The method of coordination of the nitro group is determined by the orbital energy of the electrons of the catalyst. The oxygen atom of the nitro group tends to participate in chargecontrolled interactions, and $\pi$-bond, on the contrary, requires orbitally-controlled reactions. Therefore, metals prone to charge-controlled reactions will be coordinated with the nitro group through an oxygen atom, and the metals forming orbital-controlled bonds will be combined with the $\pi$-bonds of the nitro group. The propensity to create charge-controlled bonds usually changes anabolically to the oxidationreduction potential of the metal, and the efficiency of orbitally controlled bonds increases with the oxidation-reduction potential of the elements.
In the VIII group, the redox potentials have the following values, eV.

$$
\begin{aligned}
& \mathrm{Fe}(-0.44)<\mathrm{Co}(-0.28)<\mathrm{Ni}(-0.25)<\mathrm{Ru}(0.79)< \\
& \text { Os }(0.87)<\mathrm{Rh}(0.87)<\mathrm{Pd}(0.89)<\operatorname{Ir}(0.93)<\mathrm{Pt} \\
& (0.98)
\end{aligned}
$$

Consequently, the contribution of the orbitally-controlled interaction in this series increases from left to right, and the share of the chargecontrolled interaction increases from right to left. So, on the $\pi$-route, the catalyst activates the nitro compounds, reacting with the $\pi$-bonds of the nitro group. Hence, such a path is chosen by catalysts prone to the formation of $\pi$-bonds. In the periodic table of D.I. Mendeleev ability to create $\pi$-bonds increases from right to left and from top to bottom. If we take into account the requirements of the first stage, then we can assume that metals of the type Pt, Pd and Rh, located at the bottom of the VIII-group of the Periodic Table of D.I. Mendeleev will choose the $\pi$-route.

Further selection of catalysts is done based on the transition state of the next stage. A rough estimate shows that the metals of the VIII-group 
and the I-subgroup of the periodic table Mendeleyev in the hydrogenation of nitro compounds have the following configurations (Table 1). Hence it can be assumed that $\mathrm{Pd}$ and $\mathrm{Cu}$ act by the free orbital with a high $\mathrm{s}^{0}$ character, and the remaining metals react by vacant orbitals with an increased $\mathrm{d}^{0}$ contribution.

Table 1: Electronic configurations of metals of VIII group and I-subgroup of the D.I. Mendeleyev Periodic system

\begin{tabular}{cc}
\hline Metal & Electronic configurations \\
\hline $\mathrm{Fe}$ & $3 \mathrm{~d}^{6} 4 \mathrm{~s}^{2}$ \\
$\mathrm{Co}$ & $3 \mathrm{~d}^{7} 4 \mathrm{~s}^{2}$ \\
$\mathrm{Ni}$ & $3 \mathrm{~d}^{8} 4 \mathrm{~s}^{2}$ \\
$\mathrm{Cu}$ & $3 \mathrm{~d}^{10} 4 \mathrm{~s}^{1}$ \\
$\mathrm{Ru}$ & $4 \mathrm{~d}^{7} 5 \mathrm{~s}^{1}$ \\
$\mathrm{Rh}$ & $4 \mathrm{~d}^{8} 5 \mathrm{~s}^{1}$ \\
$\mathrm{Pd}$ & $4 \mathrm{~d}^{10}$ \\
$\mathrm{Os}$ & $5 \mathrm{~d}^{6} 6 \mathrm{~s}^{2}$ \\
$\mathrm{Ir}$ & $5 \mathrm{~d}^{7} 6 \mathrm{~s}^{2}$ \\
$\mathrm{Pt}$ & $5 \mathrm{~d}^{9} 6 \mathrm{~s}^{1}$ \\
\hline
\end{tabular}

The orbital diagram shows that the metals acting by the free $s^{0}$ orbit create a symmetry inhibition at the stage of the reaction of the nitro compound with the hydrogen atom. Metals that work with a vacant $d^{0}$ orbit, on the contrary, help the reaction of the nitro group with the hydrogen atom.

At the next stage of the $\pi$-route, the hemihydrogenated form of the nitro compounds adds the second hydrogen atom. Then there is a non-catalytic reaction of the splitting of water from the intermediate product molecule and the formation of nitrosobenzene. After this stage nitrosobenzene $(\mathrm{R}-\mathrm{N}=\mathrm{O})$ enters the catalytic cycle of the $\pi$-route. Nitrosobenzene is also a sufficiently polar molecule and can be coordinated through the $\pi$-bonds of $\mathrm{N}-\mathrm{O}$ and the oxygen atom. In the $\pi$-route, nitrosobenzene is activated by the formation of $\pi$-bonds with the catalyst. Nitrosobenzene joins the second hydrogen molecule in the same orbital scheme as nitrobenzene. Here, the lowest free d-orbitals of the working catalysts also differ in favorable nodal symmetry. Catalysts acting by their vacant orbitals with a high s-character create a barrier to the hydrogenation of nitrosobenzene to phenylhydroxylamine. After the absorption of the second hydrogen molecule, phenylhydroxylamine enters the reaction. Metals that prefer charge-controlled coordination will interact with phenylhydraxylamine through an oxygen atom. The contribution of o-cordination will increase with decreasing oxidation-reduction potential of the complexing agent. Elements prone to orbitally controlled binding will adsorb phenylhydroxylamine through a nitrogen atom. And at these stages of the $\pi$-route, the catalysts acting better with free orbitals with increased d-contribution are better.

The $\pi$-route ends with the reaction of hydrogenation of phenylhydroxylamine to aniline. It can be expected that the proportion of the $\pi$-route will increase with the increase in the acceptor properties of the substituents of the nitro compounds.

Forecasts of the orbital theory of catalysis agree well with experimental data on the hydrogenation of nitro compounds on $\mathrm{Pt}, \mathrm{Pd}$, and Rh-catalysts. In a water-alcoholic medium, nitro compounds on such catalysts are hydrogenated to zero order without the accumulation of intermediates, and the reaction rate positively reacts to an increase in the acceptor capacity of the substituents. For $\pi$-route, the catalyst basicity condition is unfavorable.

For the o-route, on the contrary, the rate increases with the enhancement of the basicity of the catalyst. The o-route begins with the activation of hydrogen. The o-route at this stage puts forward the same orbital requirements as the $\pi$-route. However, already the second stage of the O-route presents absolutely opposite requirements. For o-coordination of nitrocompounds, nitroso compounds and phenylhydroxylamine, metals preferring chargecontrolled interactions, i.e., having a low oxidationreduction potential are needed. Therefore, for the o-route, catalysts from the upper part of the VIII-group of the Periodic Table of D.I. Mendeleev are needed.

In 3, 4 stages, nitrosobenzene is formed by sequential addition of two atoms of coordinated hydrogen from nitrobenzene. Orbital diagram shows that on the o-route in a more favorable position are the metals acting with free orbitals with a high s-character.

The reaction of hydrogenation of nitrosobenzene to phenylhydroxylamine continues the previous stage and presents similar requirements to the catalyst. For the hydrogenation of nitrosobenzene, catalysts that have in the coordinated state free orbitals with a high s-contribution are needed. 
For the final stage of the o-route, in which phenylhydroxylamine is converted to aniline, catalysts that release the orbitals with increased d-contribution under the influence of reagents (and also solvent, carrier) are more suitable.

So, the o-route sets absolutely opposite conditions for the catalysts than the $\pi$-route. In this way, the reaction is carried out by catalysts preferring a charge-controlled combination, necessary for o-coordination, and acting by free orbitals with a high s-character required to provide reactions between hydrogen atoms and o-coordinated nitrobenzene and nitrosobenzene.

The electronic structure of the nitro group is as follows: thirteen valence electrons of the $\mathrm{NO}_{2}$ molecule form the following configuration: $\sigma^{2}, \sigma^{2}{ }^{2}$, $\pi^{2}{ }, 2 p^{2}, 2 p^{2}, \pi^{2}{ }_{y}, \sigma_{z}^{1}$. The first three orbitals: $\sigma_{s}^{2}, \sigma^{2}{ }^{\prime}$, $\pi_{y}^{2}$ are binding, the other $-2 p^{2}, 2 p^{2}, \pi_{y}^{2}, \sigma_{z}^{1}$ remain unbound. The total number of binding electrons is six, which corresponds to an average bond order of 1.5. The molecule has an angular shape with $\pi$-bonds.

At the stage of coordinating the oxygen atom of the nitro group, nitrosobenzene is formed by successive addition of two coordinated hydrogen atoms from nitrobenzene. The orbital diagram shows that in a more advantageous position, there are metals acting by means of free orbitals with a high $\mathrm{s}$-character. The nodal symmetry of the d-orbitals creates an energy barrier at the stage of addition of the first hydrogen atom to the nitrogen atom of nitrobenzene. Slightly in a better position than the s-orbitals, there are d-orbitals during the addition of the second hydrogen atom to the hemihydrogenated form of nitrobenzene. On d-metals, the second hydrogen atom attacks the bonded portion of the oxygen atom, and on the s-metals it does not. In the latter case, the reaction occurs due to the presence of an unshared pair of oxygen atoms. A busy, unshared oxygen orbit ensures the flow of this stage and reduces the energy barrier. Thus, the orbital consideration of the hydrogenation stage of nitrobenzene to nitrosobenzene shows that the activation energy of the reaction is less for metals operating with free orbitals with a high s-character. Such a mechanism is quite the opposite of the $\pi$-coordination of the nitro group on $\mathrm{Pd}$.
The next stage of hydrogenation of nitrosobenzene to phenylhydroxylamine is similar to the previous one. On the orbital diagram, to prove this conclusion, the operating orbitals of nitrosobenzene, hydrogen and the catalyst are shown at the stage of addition of the first hydrogen atom to nitrosobenzene and operating orbitals at the stage of addition of the second hydrogen atom to the hemihydrogenated form of nitro-zobenzene. At this stage nitrosobenzene passes into phenylhydroxylamine.

Hydrogenation of the coordinated phenylhydroxylamine consists in the hydrogenolysis of the O-N bond to form an amine and a hydroxyl group. Then the hydroxyl group attaches the second hydrogen atom and turns into water. Orbital consideration also satisfactorily explains this stage of the process.

Thus, the mechanism proposed by Y.A. Dorfman, based on orbital modeling, helps to understand the complex nature of the action of catalysts in the hydrogenation of nitrobenzene and its derivatives and can be used in predicting the properties of catalysts and developing selective catalytic systems.

\section{CONCLUSION}

The classical scheme of the mechanism of hydrogenation of Haber-Lukashevich, specified and updated by V.P. Shmonina, was recognized by most authors. In most of the works, the classical scheme of the mechanism of the hydrogenation of HaberLukashevich nitrobenzene is confirmed and detailed by experimental methods. However, there are alternative judgments, as an opinion of E. Gelder et al.,

According to the mechanism of Ya.A. Dorfman, the most important factors are the electronic configurations and energies of the free orbital and the occupied orbitals of the ground state of the active catalyst, the $\sigma$ - and $\pi$-acceptor and $\sigma$ and $\pi$-donor capacity of substituents, the $\mathrm{pH}$ of the medium, which regulate the direction of chemical transformations of nitro compounds.

The complexity of studying the mechanism of nitro compounds reduction can be explained by the 
multistage nature of the reduction of the nitro group, as well as the effect on the rate of hydrogenation of many factors.

The described schemes of the mechanism for the reduction of aromatic nitro compounds allow us to consider the process from various sides, and, not contradicting each other, supplement the overall picture.

\section{ACKNOWLEDGEMENT}

We would like to express sincere gratitude to the Ministry of Science and Education of the Republic of Kazakhstan for grant financing (Project 2018-2020).

\section{Conflict of interest}

Conflict of interest declared none.

\section{REFERENCES}

1. Jüngers, J.; Sazhius, L., Kinetic methods for the study of chemical processes, L.: Chemistry., 1972, 421.

2. Aubakirov, Y.A.; Sassykova, L.R., Selective catalytic reduction of aromatic nitro compounds and hydrocarbons, Almaty, Qazaq university., 2018, 338.

3. Sokolsky, D.V.; Drouz, V.A., Reports of the Academy of Sciences of the USSR., 1950, 73(5), 949-950.

4. Shmonina, V.P.;Tarasova, D.V .; Alekseeva, G.K; Serazetdinova, V.A, Proceedings of the Institute of Chemical Sciences, Academy of Sciences of the Kazakh SSR., 1952, 8, 64-72.

5. Lukashevich, O.V., Journal of General Chemistry., 1937, 7, 2209-2213.

6. Shmonina, V.P.; Sokolsky, D.V., Scientific notes of the Kazakh State University, Almaty, 1956, 22(21), 33-42.

7. Abdrakhmanova, R.M.; Shmonina, VP.; Sokolsky, D.V., In the collection: Catalytic Reduction and Hydrogenation, Ivanovo., 1970, 46-51.

8. Shmonina, V.P.; Seredenko, V.V.; Kulazhanov, K.S. In the collection: Applied and Theoretical Chemistry, Alma-Ata., 1973, 4, 339 - 346.

9. Tyomkin, O.N., Catalytic Chemistry, M.: Chemistry., 1996, 438.

10. Rinecker, R., Proceedings of the IV International Conference on Catalysis, M.: Nauka., 1970, 2, 19 - 25.

11. Sklyarov, A.V., In the collection: Problems of kinetics and catalysis, M.: Nauka., 1975, 238-260.

12. Pernoud, L.; Candy, J.P.; Didillon, B.; Jacquot, R.; Basset, J.M., Studies in Surface Science and Catalysis., 2000, 130, 2057 - 2062.

13. Klyuev, M.V., Catalytic synthesis of amines by hydrogenation and hydroamination, Dissertation of Dr. of Chemical Sciences, M., 1991, 368.
14. Bogdanovsky, G.A.; Shcherev, G.I., Electrochemistry., 1970, 6, 318 - 322.

15. Grishina, T.M.; Logacheva, L.I.; Fadeeva, V.I.; Stratiev, A.I.; Vovchenko, G.D., Moscow State University Bulletin, Chemical Series., 1973, 5, 586-590.

16. Krasnova, N.N.; Khomchenko, G.P.; Vasilyev, Yu.B., Electrochemistry., 1975, 11(8), 1205-1209.

17. WiJngaarden, K.R. Westerterp. Industrial catalysis. Optimizing catalysts and processes, Willey-VCH VerlagGMbh\&KGaA, Wenheim, Germany., 2006, 520.

18. Babneev, A.D., Hydrogenation of organic compounds at the polarization overlay, Dissertation of a Candidate of Chemical Sciences, Ivanovo., 1968, 185.

19. Adamson, A., Physical chemistry of surfaces, M.: Mir., 1979, 568.

20. Fasman, A.B.; Sokolsky, D.V., Structure and physico-chemical properties of skeletal catalysts, Alma-Ata, Nauka., 1968, 176.

21. Sokolskaya, A.M.; Omarkulov, T.O.; Bizhanov, F.B.; Kamalov, M.Z., Reports of the Academy of Sciences of the USSR., 1972, 205(1), 107-109.

22. Dinassylova, Sh.D., Potentiometric method under pressure studies of suspended catalysts, Dissertation of the candidate of chemical sciences, Alma-Ata., 1984, 243.

23. Savchenko, V.I.; Denisenko, T.V.; Sklyar, S.I.; Simonov, D.V., Journal of Organic Chemistry., 1975, 10, 2149-2153.

24. Simonov, V.D.; Denisenko, T.V.; Savchenko, V.I.; Sklyar, S.Ya., Journal of Organic Chemistry., 1976, 12(5), 1036.

25. Vasilyev, Yu. B.; Bagotzsky, V. S.; Khazova, O. A.; Krasnova, N. N.; Sergeeva, T. A.; Bogdanovich, V. B., Elektrochim. Acta., 1981, 26(4), 545-562. 
26. Vasilyev, Yu. B.; Bagotzsky, V. S.; Khazova, O. A.; Yastrebova, T. N.; Sergeeva, T. A.; Elektrochim. Acta., 1981, 26(4), 563-577.

27. Klyuyev, M.V.; Weinstein, E.F., Neftekhimiya., 1997, 37(5), 413-419.

28. Abdullayev, M.G., Catalytic synthesis of unsymmetrical secondary amines and intermediates for their preparation, Dissertation of the Doctor of Chemical Sciences, Ivanovo, Russia., 2005, 278.

29. Becker, G., Introduction to the electronic theory of organic reactions, M.: Mir., 1965, 250-255.

30. Chemistry of nitro and nitroso groups, Ed. Fuyer, M.: Mir., 1972, 1, 34.

31. Gelder, E.A.; Jackson, S. D.; Lok, C.M., Chem. Comm., 2005, 522-524.

32. Kochetova, L. B., Kinetic regularities and mechanisms of amidation reactions, Dissertation of the Doctor of Chemical Sciences, Ivanovo, Russia., 2017, 355.

33. Gelder, E. A., The hydrogenation of nitrobenzene over metal catalysts, PhD Dissertation, Glasgow., 2005, 312.

34. Corma, A.; Concepción, P.;Serna, P., Angewandte Chemie., 2007, 46(38), 7266-7269.

35. Tereshko, L. V., Synthesis of aromatic and fatty-aromatic amines on palladium-containing catalysts, Dissertation of Candidate of Chemical Sciences, Ivanovo, Russia., 1990, 163.

36. Kochetova, L. B.; Klyuyev, M. V.; Zhurnal obshchey khimii., 2008, 78(2), 286-287.

37. Kochetova, L. B.; Klyuyev, M. V., Neftekhimiya, 1997, 37(5), 420-426.

38. Sassykova, L.R.; Sendilvelan, S.; Otzhan, U.N.; Vassilina, G. K.; Zhumakanova, A. S.; Mataeva, Z.T.; Amangeldi, M. B.; Abildin T.S., News of the National Academy of Sciences of the Republic of Kazakhstan, Series of Geology and Technical Sciences., 2018, 428(2), 18-28.

39. Popova, N. M .; Babenkova, L. V .; Savelieva, G. A., Adsorption and interaction of the simplest gases with metals of the eighth group, AlmaAta, Science of the Kazakh SSR., 1979, 280.

40. Efremenko, I., J. Mol. Catal. A: Chemical., 2001, 173(1-2), 19-59.

41. Cárdenas-Lizana, F. The development of supported gold catalysts for selective hydrogenation applications: PhD Dissertation, Heriot-Watt University., 2009, 258.
42. Shrikant, L. K.; Rajeev, A., R., Ind. Eng. Chem. Res., 1987, 26(9), 1746-1750.

43. Dorfman, Ya.A., Liquid-phase catalysis. Orbital modeling, Almaty, Nauka, Kazakh SSR., 1981, 364.

44. Catalytic liquid-phase reduction of aromatic nitro compounds. Ed. Ovchinnikov, P.N., Leningrad., 1969, 210.

45. Lefedova, O.V.; Gostikin, V.P.; Ulitin, M.V., Zhurnal prikladnoy khimii., 1993, 66(3), 556-561.

46. Lefedova, O.V., Selective liquid-phase catalytic hydrogenation of substituted nitroand azobenzenes, Dissertation of the doctor of Chemical Sciences, Ivanovo., 2002, 352.

47. Denisenko, T.V.; Savchenko, V.I.; Simonov, V.D.; Sklyar, S.Ya., Zhurn. org. khimii., 1982, 18, 1498-1503.

48. Vorozhtsov, N.N., Fundamentals of synthesis of intermediate products and dyes, M., Goskhimizdat., 1955, 750.

49. Gostikin, V.P., In Collection: Questions of kinetics and catalysis, Ivanovo., 1985, 3-9.

50. Litvin, E.F., Kozlova, L.M.; Shapiro, A.B., In collection: Catalytic reactions in the liquid phase, Alma-Ata., 1974, 75-77.

51. Klopman, G., Reactivity and chemical reaction pathways, M., Mir., 1977, 227.

52. Sassykova, L.R., Catalytic reduction of monoand dinitro compounds of the aromatic series, Dissertation of the Candidate of Chemical Sciences, Kazakhstan, Almaty., 1996, 223.

53. Sassykova, L.R.; Otzhan, U.N.; Kurmansitova, A.K.; Serikkanov, A.A.; Aubakirov, Y.A.; Zhumakanova, A.S.; Kenzhebekov, A.S., News of the National Academy of Sciences of the Republic of Kazakhstan, Series of Chemistry and Technology., 2017, 422(2), 147-156.

54. Baiseitov, D.A.; Gabdrashova, Sh.E.; Akylbai, A.K.; Dalelkhanuly, O.; Kudyarova, Zh.B.; Sassykova, L.R.; Tulepov, M.I.; Mansurov, Z.A., Int. J. Chem. Sci., 2016, 14(1), 261-268.

55. Baiseitov, D.A.; Tulepov, M.I.; Sassykova, L.R.; Gabdrashova, Sh.E.; Magazova, A.N.; Dalelkhanuly, O.; Kudyarova, Zh.B.; Mansurov, Z.A. Bulgarian Chemical Communications., 2017, 49(3), 600-607.

56. D.A. Baiseitov, Sh.E. Gabdrashova, A.N Magazova, O.Dalelkhanuly, Zh.B.Kudyarova, M.I. Tulepov, L.R. Sassykova, Z.A. Mansurov, Int. J. Chem. Sci., 2016, 14(1), 244-250. 
57. Tulepov, M. I.; Mansurov, Z. A.; Kazakov, Y. V.; Abdrakova, F. Y.; Sultanova, Z. L.; Rakhova, N. M.; Madiyev, S. S.; Golovchenko, O. Y.; Sassykova, L. R.; Tolep, D. M.; Chikhradze, N.; Chikhradze, M. N., Orient J Chem., 2018, 34(6), 3037-3043.

58. Sassykova, L.R., Chemical and biochemical engineering quarterly, 2017, 31(4), 447-453

59. Sassykova, L.; Aubakirov, Y., Chiang Mai J. Sci., 2018, 45(1), 474-483.

60. Lefedova, O.V.; Gostikin, V.P.; Ulitin, M.V., Izv. VUZov, Khimiya I Khim. Tekhnologiya., 1991, 34(7), 75-78.

61. Klyuev, M.V., Hydrogenation amination of carbonyl compounds, Dissertation of the Candidate of Chemical Sciences, USSR, Chernogolovka., 1979, 208.

62. Aubakirov, Y.; Sassykova, L.; Sendilvelan, S.; Bhaskar, K.; Otzhan, U.; Amangeldi, M.; Abildin, T.; Zhumakanova, A.; Zhussupova, A.; Zharkyn, M., Journal of Chemical Technology \& Metallurgy., 2019, 54(2).

63. Baiseitov, D.A.; Gabdrashova, Sh.E.; Magazova, A.N.; Dalelkhanuly, O.; Kudyarova, Zh.B.; Sassykova, L.R.,; Tulepov, M.I.; Mansurov,
Z.A.; Dalton, A.B., International Journal of Biology and Chemistry., 2015, 8(2), 66-70.

64. Otzhan, U.N.; Kurmansitova, A.K.; Sassykova, L.R.; Serikkanov, A.A.; Kenzhebekov, A.S.; Starikov, E.B., International Journal of Biology and Chemistry., 2016, 9(2), 40-44.

65. Finkel'shteyn, A.B.; Kuz'mina, Z.M.; Zhurnal fizicheskoy khimii, 1966, 40(1), 166-169

66. Finkel'shteyn, A.B.; Kuz'mina, Z.M.; Doklady AN SSSR., 1964, 158(1), 176-178.

67. Kathirvelu, B.; Subramanian, S.; Govindan, N.; Santhanam, S., Sustainable Environment Research., 2017, 27(6), 283-290.

68. Bhaskar, K.; Sassykova, L.R.; Prabhahar, M.; Sendilvelan, S., International Journal of Mechanicaland Production Engineering Research and Development., 2017, 8(1),399-406.

69. Yao, H.C.; Emmett, P.H., Journal. of the American Chemical Society., 1959, 81(16), 4125-4132.

70. Hernandez, L.; Nord, F.F., Journal.Coll.Sci., 1948, 3, 363-375.

71. Kazuo, T., Sci. papers.Inst.Phys.and Chem. Res., 1962, 56(4), 285. 